Voix et Images

voixetimages

\title{
Sur Jean Éthier-Blais
}

\section{Robert Major}

Volume 24, numéro 3 (72), printemps 1999

La littérature québécoise sous le regard de l’autre

URI : https://id.erudit.org/iderudit/201452ar

DOI : https://doi.org/10.7202/201452ar

Aller au sommaire du numéro

\section{Éditeur(s)}

Université du Québec à Montréal

\section{ISSN}

0318-9201 (imprimé)

1705-933X (numérique)

Découvrir la revue

\section{Citer cet article}

Major, R. (1999). Sur Jean Éthier-Blais. Voix et Images, 24(3), 589-598.

https://doi.org/10.7202/201452ar d'utilisation que vous pouvez consulter en ligne.

https://apropos.erudit.org/fr/usagers/politique-dutilisation/ 


\section{Essai}

\section{Sur Jean Éthier-Blais}

\section{Robert Major, Université d'Ottawa}

Jean Éthier-Blais, on le sait, est mort le 12 décembre 1995, soudainement, en pleine rue, sortant tout juste d'une réception, littéraire et amicale, et en route vers une seconde, littéraire et internationale. Cette mort subite d'un homme en marche, entre deux fêtes marquées au coin de la littérature, de l'amitié, et des rapports internationaux, a peut-être valeur de symbole. Elle me semble conforme à l'image qu'on se fait - ou, plutôt, qu'on pourra se faire maintenant, grâce à des publications récentes d'un homme dont le destin est sans doute remarquable. Cette vie, à la regarder de loin et dans ses repères schématiques, incarne quelques aspects fondamentaux de notre destin collectif. Elle réunit en faisceau toute une série de forces sans doute contradictoires ou du moins hétérogènes, mais dont Éthier-Blais a fait harmonie.

Natif d'une petite ville nordique, essentiellement forestière, aux marches du royaume, fils d'un homme tôt disparu mais qui semble, dans le souvenir de l'auteur, prendre les formes de quelque héros sorti tout droit des Engagés du grand portage, goûtant très tôt à l'amer fruit qu'on offre en partage aux minoritaires et aux citoyens de seconde catégorie, Jean Éthier-Blais se nourrira d'un héritage qui, dans un autre registre et dans un autre lieu, nous a donné un Menaud. Mais ce Menaud aura aussi connu les livres et la culture. La grande culture humaniste, européenne, c'est-à-dire grecque, latine, française, anglaise et allemande, dont il aura fait sa deuxième patrie et l'aune à laquelle il mesurera ce que ses compatriotes lui donneront à lire et ce qu'il pourra lui-même écrire. Ces deux faces du Canada français ou du Québec, comme on voudra et comme il l'a lui-même voulu vers la fin de sa vie - sont présentes en lui et dans son œuvre: enracinement, sentiment d'appartenance, fidélité aux origines, mais ouverture, par la culture, au monde et à son infinie richesse. Se lovant dans les livres, Jean Éthier-Blais sera, néanmoins, homme de combat; de multiples combats: pour les siens, pour les écrivains, pour la dignité humaine. Il lui sied d'être mort en marche.

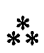

Les auteurs, c'est bien connu, peuvent mourir deux fois: la première, comme personnes, la seconde comme auteurs. Quelquefois l'ordre des deux morts est inversé. Combien d'écrivains n'ont guère survécu à leur mise en bière! Leurs livres retournent en poussière, suivant de peu la 
dépouille mortelle. Un infime pourcentage trouve place dans les manuels ou les histoires de la littérature; un nombre plus petit encore sur les rayons des bibliothèques ou dans la ferveur des lecteurs. Si l'on en croit les sociologues de la littérature, tout se joue dans les dix années qui suivent la mort physique.

Mais les conditions de survivance restent mystérieuses, peu étudiées, souvent déroutantes. Et les cas de renaissance sont nombreux. Quelquefois cela tient à un fil, au hasard d'une rencontre, à une conjoncture favorable. Mais comment définir une "conjoncture"? Combien d'auteurs ont été irrémédiablement perdus par les hasards d'un sort capricieux: l'incendie d'une bibliothèque monastique, une invasion barbare, la cécité de contemporains? Que serait Nelligan n'eût été de Louis Dantin? Vraisemblablement une note infrapaginale et quelques poèmes épars, miraculeusement rescapés. Il ne serait pas une œuvre. Peut-être suffit-il, en effet, d'un premier cercle de lecteurs fervents ou d'amis fidèles. Ou d'une seule personne.

Le lancement du premier numéro des Cabiers Étbier-Blais ${ }^{1}$, faisant suite à la publication récente de deux volumes d'essais et d'études sur cet auteur (Jean Éthier-Blais: une vie en écriture ${ }^{2}$; Jean Éthier-Blais: dictionnaire de lui-même $e^{3}$ ), incite à nous pencher quelque peu sur le phénomène. Celui-ci dépasse nettement ce qui se produit au décès ou au moment du départ à la retraite d'un universitaire, lorsque ses collègues et amis lui présentent un recueil d'hommages, façon de témoigner de la contribution de ce dernier à son champ d'intérêt ou à la formation de jeunes chercheurs. De tels volumes sont toujours mérités, assez souvent intéressants. Il sont quasi toujours hétéroclites. Cela n'est pas forcément un défaut. Car si, le plus souvent, la gerbe d'hommages est composée en fonction des intérêts des participants, intérêts quelquefois fort éloignés de ceux du destinataire, la conjonction circonstancielle de ces intérêts divergents postule néanmoins la richesse de leur point d'origine (le jubilaire) et la féconde diversité du monde auquel celui-ci appartenait.

Or, dans le cas qui nous intéresse présentement, Jean Éthier-Blais n'est pas le prétexte de quelques recueils quelque peu disparates. Il est l'objet d'un travail collectif et d'un mouvement assez large. C'est l'écrivain, le romancier, le poète, l'essayiste, le mémorialiste, le chroniqueur, le critique littéraire et le critique d'art, en somme l'auteur protéiforme d'une œuvre considérable, qui est soumis à l'examen sous un multiple éclairage. L'homme aussi, et peut-être surtout.

Je ne sais si l'équivalent d'un tel florilège, à la fois aussi rapide et abondant, existe dans notre littérature. Le phénomène des Cabiers est assez connu en France, certes, où de nombreux écrivains et intellectuels sont l'objet de l'attention d'un cercle d'amis ou de fervents admirateurs, ou encore de chercheurs qui se penchent sur l'œuvre pour assurer la publication des inédits et la diffusion de tout ce qui peut concerner l'étude de l'auteur: chronologie, témoignages, bibliographies, documents iconographiques, etc. Au Québec, le cas est beaucoup plus rare, sinon inédit. À la fin des années cinquante, Robert Choquette fut certainement le 
premier des auteurs ainsi privilégiés. Entre 1956 et 1964, le Collège de Saint-Laurent publia neuf numéros des Cabiers $d u$ Cercle Robert Choquette. Mais ces cahiers portent le nom de l'auteur, sans être pour autant consacrés à son œuvre. Plus récemment, on vit l'émergence de "Cahiers Jacques Ferron", "Cahiers Gabrielle Roy ", "Cahiers Louis Dantin", qui sont à vrai dire les noms donnés à des collections. Chacune de ces collections semble avoir pour ambition de publier, sous la forme de volumes portant des titres distincts, soit des œuvres inédites de l'écrivain éponyme, soit des travaux sur lui.

Les Cabiers Êthier-Blais sont d'une autre nature. Il s'agit d'une publication annuelle qui contiendra, ainsi que l'indique son sous-titre programmatique ("Documents Essais - Outils - Témoignages"), une diversité de matériaux portant sur Jean Éthier-Blais. Le premier numéro présente, outre une introduction de son directeur, Martin Doré, un certain nombre de pièces intéressantes: des lettres envoyées à des amis français, les Paupert, entre 1951 et 1980; un bref entretien où ÉthierBlais commente sa poésie, ses romans et exprime son admiration pour Lionel Groulx; un long commentaire sur Les pays étrangers par Martin Doré ; un émouvant témoignage d'une collaboratrice et amie, Laure Belleau; les confidences enjouées et chaleureuses d'une autre amie, la peintre Marcelle Ferron (dont l'une des toiles orne la couverture des Cabiers); un supplément à la chronologie déjà entamée dans Jean Êthier-Blais. Une vie en écriture; des bibliographies complémentaires à celles publiées dans le même volume; un index des œuvres mentionnées dans les articles du Devoir (1960-1989). Ces "outils" ont été réalisés par l'animateur de l'entreprise, Martin Doré. Un certain nombre de "notes de lectures" complètent le tout. Ces notes étonnent à première vue, car elles portent sur une diversité d'auteurs ou de sujets (Gaston Miron, Jacques Ferron, Gabrielle Roy, histoires et manuels littéraires), mais on s'aperçoit rapidement que le directeur de la revue les signe presque toutes et que chaque recension établit un lien précis avec Éthier-Blais, si ce n'est que pour déplorer les silences à son endroit dans tel ou tel volume.

Dans sa présentation, Martin Doré décrit les Cabiers comme un lieu:

Dans un premier temps, il nous paraît donc nécessaire de créer un lieu où tous ceux qui - écrivains, essayistes, critiques veulent s'interroger sur l'œuvre d'Éthier-Blais, sur l'homme, et sur les rapports que l'un et l'autre établirent avec leur temps, puissent trouver une occasion de publier le fruit de leur réflexion. Mais, et de façon tout aussi importante, ce lieu doit permettre de rassembler et de publier ce qui n'est pas encore connu de cette œuvre, dont la correspondance. (p. 3)

Avec ce premier numéro, le lieu se peuple et se meuble, et on a le goût d'y séjourner. Les portraits de l'homme nous mettent en présence d'un être complexe et attirant, fort loin des caricatures déjà dessinées; la correspondance éclaire une œuvre et une époque, et la nature des liens à la fois amicaux et spirituels entre les êtres; les documents sont rigoureusement présentés et seront fort utiles 
aux chercheurs. En somme, cette première réalisation augure bien des Cabiers Éthier-Blais à venir.

Ce premier Cabier, de fait, est dans le prolongement immédiat du volume Jean Éthier-Blais. Une vie en écriture 1925-1995. Celui-ci contenait déjà treize témoignages sur l'homme, choisis soigneusement par le responsable du volume, Martin Doré, pour rendre compte des différentes facettes de la vie de Jean Éthier-Blais :

J'ai choisi les collaborateurs de ce livre selon des principes simples. D'abord, ils devaient être écrivains ou rompus à l'écriture. J'avais choisi chacun pour qu'il parle d'une partie précise de la vie d'Éthier-Blais, quitte à déborder dans la suite. Je voulais en effet suggérer l'étendue de cette vie dans le temps, l'espace et l'activité. Jean Éthier-Blais naquit en Ontario, fit ses études à Montréal, à Paris et à Munich; il vécut en Amérique, en Europe, en Afrique et en Asie; il fut diplomate, professeur, universitaire, critique et écrivain; il s'impliqua dans la défense des écrivains et dans l'organisation de manifestations d'écrivains. Je voyais ce livre comme une sorte de biographie, forcément lacunaire, où l'homme apparaîtrait à travers l'idée que ses amis s'en font. (p. 17)

Le choix a été judicieusement fait. Chacun des répondants livre un texte d'une belle qualité, avec peu de recoupements. Les plus longs constituent un portrait remarquable de l'homme que fut Éthier-Blais, mais aussi de son époque et des milieux qu'il a fréquentés. De nombreux lecteurs seront étonnés de découvrir un Jean Éthier-Blais que la lecture de ses œuvres ou de ses chroniques journalistiques ne laissait pas deviner.
L'égotiste, le fantaisiste, l'ironiste et, disons-le, le quasi-dandy imprégné de snobisme, s'estompe pour être remplacé par l'homme de cœur, l'homme de courage, l'intellectuel actif n'hésitant pas devant toutes sortes d'engagements exemplaires.

Dans le détail, cela donne de nombreux portraits, assez contrastés mais jamais contradictoires. Ainsi Guy Lafond et Robert Vigneault parlent des années passées au Collège de Sudbury, mais sous des angles différents et complémentaires: Guy Lafond insistant sur l'amitié et lès liens avec François Hertel, Robert Vigneault sur l'héritage de la formation classique et les rapports d'ÉthierBlais avec la formation reçue. Catherine et Jean-Marie Paupert racontent les années parisiennes, mais sous des angles particuliers: celle-là s'attardant quelque peu à l'aspect bohème de la bande d'amis, celui-ci à la vie communautaire au Centre International d'étudiants de L'EauVive. Moënis C. Taha-Hussein évoque brièvement leurs vies d' élèves étrangers" à l'École Normale Supérieure de la rue d'Ulm, alors que Yerri Kempf se rappelle leur culte commun de la langue française et l'attitude peu "québécoise" d'Éthier-Blais, Ontarien de naissance et donc traitant d'égal à égal avec les anglophones, et dans leur château fort de McGill en plus. Jean-Pierre Duquette, pour sa part, livre un texte remarquable sur "trois moments " à cette même université : la grève menée par Éthier-Blais et cinq collègues pour protester contre l'emprisonnement de Gaston Miron et d'autres écrivains québécois en octobre 1970; le difficile passage à la direction du Département de langue et littérature françaises de 1971 à 
1973; et le don de sa bibliothèque québécoise que fit Éthier-Blais au Centre d'études québécoises à l'Université de Bologne. "Je veux que la littérature de mon pays soit connue", disait-il alors, joignant le geste généreux aux bonnes intentions. Michel Gaulin peint en termes évocateurs l'amitié d'un "admirable aîné" qui couvre les dix dernières années de la vie d'Éthier-Blais, alors que Claude Michel Cluny se remémore une commune fascination pour l'Orient et une amitié littéraire d'abord scellée en Tunisie. Deux textes décrivent tout particulièrement l'homme engagé: René le Clère raconte de savoureuses anecdotes rattachées aux responsabilités assumées par ÉthierBlais au sein du PEN, de 1980 à 1989, couronnées par la coprésidence du $54^{\mathrm{e}}$ Congrès mondial du PEN International à Toronto et Montréal; par ailleurs, Jean-Marc Léger fait état des nombreux services rendus par ÉthierBlais comme président de la Fondation et du Centre de recherche Lionel-Groulx dans les dernières années de sa vie. Un texte de Martin Doré clôt la section des témoignages et, en quelque sorte, boucle la boucle. En effet, il y raconte son unique rencontre avec Éthier-Blais et les premiers pas dans cette entreprise d'exploration systématique de l'œuvre et de l'homme qui sera maintenant menée en l'absence du premier intéressé.

La deuxième moitié du volume est consacrée à un choix de lettres représentatives tirées de la correspondance d'Éthier-Blais et à un certain nombre de documents fort utiles et rigoureusement présentés (chronologie, bibliographies, index), le tout préparé et présenté par Martin Doré.
Le même, l'année suivante, en collaboration avec Jean-Pierre Duquette, a préparé le volume Jean Éthier-Blais: Dictionnaire de luimême. Essais sur l'œuvre, fruit d'un colloque tenu à Montréal en novembre 1997 mais qui, pour des raisons obscures, n'est aucunement mentionné dans le volume lui-même. Celui-ci, comme celui qui l'a précédé et comme le Cabier, prend la forme et d'un recueil de textes et d'un instrument de travail, puisque le tiers du livre est consacré à un précieux index onomastique des articles d'Éthier-Blais publiés dans Le Devoir entre 1960 et 1989. Toutefois, à la différence du volume précédent, Jean Éthier-Blais: Dictionnaire de lui-même présente des analyses de l'œuvre elle-même.

Certes, l'homme, ses manies, son ironie, ses contradictions, ses mystères, en somme, son mode paradoxal et fascinant d'être-au-monde, n'est jamais très loin, la plupart des signataires de ces articles ayant connu Jean Éthier-Blais et pouvant facilement dépister les traces du mémorialiste et de l'autobiographe dans les œuvres les plus diverses, ou n'hésitant pas à évoquer quelque anecdote révélatrice pour introduire ou asseoir leurs propos. D'ailleurs, quelques articles, ceux de Naïm Kattan, de François Ricard, de Catherine Paupert, de Jean Royer, de Jean-Marie Paupert, de Roger Paul Gilbert sont essentiellement des témoignages sur l'homme: l'ami, le professeur, le collègue, le jeune agrégatif d'histoire, le croyant, le chroniqueur. Ce qui ne réduit pas leur intérêt, au contraire.

Mais, au bout du compte, Jean Éthier-Blais survivra grâce à la qualité 
ou à l'importance de ses textes. La ferveur, l'affection ou la reconnaissance de ses proches n'y pourront rien. Ainsi est-il heureux que de nombreux articles s'attachent à l'œuvre dans.ses multiples facettes. La préface de Jean-Pierre Duquette indique les ambitions et les limites de l'entreprise :

\begin{abstract}
les auteurs dressent un premier inventaire de l'héritage intellectuel et littéraire du poète, du romancier, de l'essayiste et du critique. [...] Ce livre vise davantage à jeter les bases de certaines pistes de lecture et d'interprétation qu'à proposer des approches définitives d'une production multiforme, tout entière élaborée sous le signe de la perfection du style. (p. 15)
\end{abstract}

Ces pistes de lecture effectuent un balisage qui est peut-être d'ores et déjà révélateur. Certaines pistes sont beaucoup plus fréquentées, traçant déjà des contours qui augurent du premier jugement porté sur l'importance relative des différentes œuvres d'Éthier-Blais. Paradoxalement, c'est surtout le chroniqueur, le critique, l'essayiste qui retient l'attention. Un seul article, mais excellent, porte sur la poésie d'Éthier-Blais. Joseph Bonenfant y pose la question: "Est-il possible que le Jean Éthier-Blais le moins ironiste, le plus profond, le plus intime soit celui qui se révèle en tant que poète, et que ce soit celui-là qu'on connaisse le moins?" (p. 197) Tout en laissant voir ce qui serait à étudier dans cette poésie ("le verset, sa forme préférée, la rythmique, les structures de l'imaginaire, le régime de l'image, la rhétorique, si importante, de l'invocation, la stylistique de l'interpellation, les actes de discours, la force illocutoire de l'énoncé, la fonction expressive du locuteur, [...] l'originalité de la métaphore "), Joseph Bonenfant s'en tient à une analyse, à travers les quatre recueils, de "l'univers spirituel de cet homme d'une culture prodigieuse" (p. 197). Empruntant en quelque sorte la position qu'était celle d'Éthier-Blais luimême devant le poète, il lui demande "la clé de son espace intérieur, l'étendue de ses mythes personnels" (p. 198). Un certain nombre de clés essentielles sont: le silence, la solitude, le combat spirituel, la présence de la mort, la création.

Ni religieux, ni antireligieux, à mi-
chemin de l'idéal païen et de
l'idéal chrétien, Éthier-Blais a été,
comme poète, un homme à l'affút
des valeurs spirituelles. Brillant
causeur, il était un homme de
silence. Invité, sollicité et entouré,
il affectionnait par-dessus tout la
solitude. Amoureux de la vie, et
pour qu'il le fût davantage, il n'a
jamais oublié qu'il devait mourir.
Maintenant qu'il est de l'autre
côté du miroir, il nous laisse des
livres comme vestiges du mystère
de son être. Sa poésie est pour
nous, plus que jamais, révélatrice
de ce mystère, parce qu'elle
s'offre désormais à notre lecture
comme une impossible, mais
réelle, totalité. (p. 204)

De même, un seul article porte sur le nouvelliste, mais par un spécialiste du genre. Michel Lord y étudie $L e$ désert blanc: chacune des nouvelles du recueil, d'abord, mais aussi le fonctionnement du recueil comme ensemble. De façon subtile et convaincante, il montre qu'Éthier-Blais est tout le contraire d'un écrivain classique: au contraire, "nous avons affaire à un écrivain hors cadre, hors norme, qui déjoue les canons de la diction et de la syntaxe du récit traditionnel " (p. 186): 
Dès lors, apparaît clairement la liberté formelle propre au discours fictionnel bref chez Éthier-Blais, chaque nouvelle épousant les formes d'un parcours d'errance, de la dérive d'un narrateur ou d'un acteur avançant dans la vie plus ou moins aveuglément à la rencontre de son destin. (p. 186)

Cette dérive, toutefois, n'est pas gratuite ni dépourvue de sens, puisqu'elle construit une vérité morale, un art de vivre, une leçon de choses d'une profonde simplicité.

Pour sa part, Laurent Mailhot étudie les deux volumes de souvenirs d'Éthier-Blais, dépistant les sortilèges de l'autobiographe ou de l'autographe. "Le Selfbook de Jean ÉthierBlais s'étend jusqu'à ses récits, ses romans, ses critiques, ses essais. Mais il est d'abord inscrit, dessiné, dans le diptyque sur l'enfance et l'adolescence." (p. 156) Cet article, tout en effectuant une analyse des motifs, des images, des thèmes récurrents (la cave, le grenier, la culture, le nom et ses prestiges), est l'occasion d'une réflexion stimulante sur les écritures du moi. Ce récit de vie est celui d'un écrivain et d'un lettré. Laurent Mailhot excelle, d'ailleurs, à suivre à la trace l'homme de culture qu'était Éthier-Blais, relevant les références, les allusions, en somme, l'érudition, multipliant lui-même les allusions et les notes explicatives.

Un article, donc, sur le poète, le nouvelliste, l'autobiographe. Et, étonnamment, un seul également sur le romancier. Yvon Rivard y effectue un rapprochement subtil et éloquent entre Ethier-Blais et Rilke, ou plutôt entre les personnages de chacun : Malte des Cabiers de Malte Laurids Brigge de Rilke et Théodore de
Mater Europa. Les deux racontent la naissance d'un écrivain et ce que cela comporte comme aventure métaphysique, rapport aux autres et à soi :
Malte et Théodore, en bons jeunes écrivains qu'ils sont, vont d'abord écrire contre le néant et la mort, ils vont beaucoup réflé- chir avant de laisser le monde se réfléchir dans leur regard. Pour ce faire, ils devront non seulement quitter leur pays natal mais aussi renoncer à l'amour, comme si la pensée et l'écriture, l'écriture- pensée ne pouvait se cultiver que dans la plus grande solitude, celle des prêtres-professeurs qu'admire Théodore ou celle des clochards à laquelle Malte se voit réduit. [...] l'écrivain, selon Malte et Théo- dore, se livre à la solitude comme à un exercice nécessaire en vue de parvenir à ce que Rilke ap- pelle "l'indifférence intime de son cœur". (p. 43)

Écrire comme absolu, "pour atteindre ou reconquérir cette "immobilité dans l'être" dans laquelle le narrateur de Mater Europa voit la condition même du bonheur" (p. 40).

À côté de ces articles portant sur des genres ou une œuvre particulière, quelques textes embrassent plus large, portant sur une thématique ou sur l'une ou l'autre figure emblématique: la France, "foyer intérieur de sa pensée" (Réjean Beaudoin) ou Mère (André Maindron); l'Italie, objet d'un récit de voyage (Voyage d'biver), "mère des arts et des lettres" et lieu de ressourcement (Anne de Vaucher Gravili). C'est également le cas pour la question plus générale du style, abordée par Michel Gaulin dans une brève description de "l'esthétique de l'écriture" (p. 229) chez Éthier-Blais; ou encore la question de l'ironie, 
étudiée par Jean-Claude Brochu dans le Dictionnaire de moi-même.

Par ailleurs, l'essayiste, le critique, le chroniqueur qu'était ÉthierBlais est l'objet de l'attention d'un grand nombre de collaborations. Martin Doré se livre à une description schématique de la vaste production journalistique, l'étudiant à la lumière du titre projeté par ÉthierBlais lui-même pour la publication intégrale de cet ensemble: "Le Miroir critique". Il l'aborde en distinguant des chapitres (la littérature, la société, le moi) et des périodes, avec leurs caractéristiques propres. Au cours de sa carrière, de 1960 à 1989, Éthier-Blais a publié 904 articles, dont seulement 66 (un maigre 7\%) ont été repris en volumes (Signets $I$, II, III). "On doit dès lors se poser une question: peut-on déduire, de la seule lecture des Signets, l'esthétique critique d'Éthier-Blais, en sachant qu'aucun article écrit après 1968 dans Le Devoir n'est repris en recueil?" (p. 219, n. 1). Poser la question, c'est peut-être y répondre. Mais en attendant l'intégrale, les autres collaborateurs au volume se livrent néanmoins à des analyses précieuses de ce qui est présentement aisément disponible de ce bloc.

Robert Vigneault, spécialiste de l'essai, trouve en Éthier-Blais "l'exemple d'un essayiste accompli ayant parcouru tout l'empan de l'essai, selon la typologie de Lukács" (p. 56), maîtrisant autant l'essai critique (celui qui interroge les œuvres pour en manifester le sens) que l'essai intime (celui qui se passe de la médiation des œuvres pour, directement, extraire la signification de la vie elle-même). Selon lui, dans ses Signets, Éthier-Blais pratique une véritable philosophie du style, se créant par l'écriture, recréant du même coup un monde et une vie stylisée:

Le Style! Ce terme, pudiquement
ignoré il n'y a pas si longtemps
comme élitiste et démodé, je le
gratifie ici de la majuscule parce
qu'il me paraît le maître-mot non
seulement de la poétique mais de
la vision du monde de Jean
Éthier-Blais. (p. 58)

Face à ces mêmes Signets, Isabelle Daunais relève, parmi les images récurrentes de l'auteur dans sa prose critique (la fuite et l'immobilité, la grandeur, l'amitié, le rêve...), une image ou idée, surtout, qui revient, s'impose, structure le regard et le jugement, celle de la bauteur de la littérature. L'écrivain, pour ÉthierBlais, doit s'installer dans la hauteur, qui est moins une position qu'une exigence de l'esprit, un détachement du quotidien, des circonstances et même du temps présent. Cette recherche de l'essentiel, cette exigence de l'absolu conduit Éthier-Blais à saisir les œuvres, québécoises ou françaises, non pas "dans la part d'éphémère, de daté ou de circonstanciel qui pourrait leur revenir; elles sont lues d'emblée dans leur durée, dans leur rapport d'identité avec toutes les autres cuvres littéraires" (p. 138). Jean-Marcel Paquette, pour sa part, dans un article quelque peu apparenté à celui d'Isabelle Daunais, se livre à un survol amusant des "passions et détestations littéraires" d'Éthier-Blais dans ses Signets. Dans ses affections littéraires comme dans ses mépris, celui-ci n'était pas toujours transparent, se permettant de louer publiquement ce qu'il n'aimait guère et blâmant quasi toujours obliquement, en quelque sorte. En 
effet, l'écrivain critiqué était moins condamné que manière de repoussoir pour mettre en valeur un écrivain admiré. Certes, il y a des têtes de Turc - Sartre, de Beauvoir, Hemingway, êtres vulgaires comme il y a des fidélités littéraires remarquables (Chateaubriand, SaintSimon, Constant, Lionel Groulx, Hertel, Morin, Nelligan...). Une dominante existe, parmi ces derniers, selon Jean-Marcel Paquette: "la marginalité par une solitude accomplie, le destin lié à une tâche, qui est l'œuvre seule" (p. 237); les écrivains aimés et toujours rappelés ont connu l'expérience de l'exil, soit réelle, soit symbolique, cette dernière se vivant à l'intérieur de la solitude de l'œuvre.

Toujours face aux Signets, dans ce cas le volume III sur les écrivains québécois, André Brochu se livre à une très belle réflexion sur "l'imaginaire dans la prose critique" d'Éthier-Blais. Ce faisant, il pose un certain nombre de questions essentielles, sur "la consistance de l'écriture et de l'œuvre d'Éthier-Blais", sur "les mérites littéraires" de l'œuvre, autant d'imagination que critique. Postulant que "seuls les vrais écrivains manifestent, à travers leurs discours, un imaginaire nourri et cohérent; et que plus riche est leur univers imaginaire, plus grande est leur réussite" (p. 6970 ), il s'arrête à deux motifs importants ("la fenêtre, l'envol et tout ce qui se greffe autour") qui non seulement structurent les textes critiques, romanesques et poétiques d'ÉthierBlais, mais que ce dernier retrouve avec volupté chez les écrivains qu'il aime. Cette première prospection de l'imaginaire critique d'Éthier-Blais est aussi pour André Brochu l'occasion de préciser en quelques phrases prégnantes la position critique d'Éthier-Blais (et sans doute, en grande partie, de Brochu lui-même): la critique comme lecture, connaissance intime, affaire de culture et de jugement, écriture "qui conjugue intelligence et sensibilité, réflexion et plaisir" (p. 70).

Tous ces articles, rédigés pour la plupart par d'excellents critiques, en pleine possession de leurs moyens, compośent un volume d'une très belle venue, équilibré, substantiel, agréable à lire. Je crois, aussi, qu'Éthier-Blais, indépendamment des plaisirs d'amour-propre ressentis à le soupeser, en aurait apprécié la tonalité d'ensemble: beaucoup de culture et d'aisance dans le propos, pas de jargon.

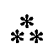

En déterminant que ses archives privées (les manuscrits, une bonne partie de sa correspondance, et son Journal, surtout, qu'il tenait religieusement) ne seraient accessibles qu'en 2025, Jean Éthier-Blais postulait vraisemblablement sa propre survie littéraire. Trente ans, cela suppose que le premier moment d'émotion est passé, avec ce qu'il aurait pu comporter de célébration factice; que la plupart des intimes auront disparu; que le temps aura fait son œuvre. Dans le monde capricieux des lettres québécoises, prompt aux impressions évanescentes, aux fugues sans lendemain et aux passions sans fondement, trente ans c'est un siècle. Qui se précipitera dans vingt-cinq ans pour prendre connaissance du journal d'Éthier-Blais - non pas pour étancher une curiosité avide, 
mais pour voir si le Québec aura eu son Saint-Simon? Au train où vont les choses, d'ailleurs, restera-t-il des francophones au pays pour s'intéresser à la question?

Apprécions l'ironie de la situation, typique du personnage. Lui, qui s'est tellement révélé dans ses écrits, à la limite ne parlant que de lui et le disant à qui voulait l'entendre, aura choisi, à la toute fin, de se dérober!

Apprécions aussi, et peut-être davantage, ce beau pari sur l'avenir. Éthier-Blais, dont une mélancolie persistante traverse les écrits et qui n'était pas à l'abri du désespoir ("Qui lira-t-on, de nous tous, dans un demi-siècle? [...] Nos efforts auront été vains. [...] Parfois, mon cœur faiblit, l'espoir me quitte et je pense qu'au milieu du vingt-et-unième siècle, nous serons des Américains comme les autres. L'Amérique n'était pas digne du français ${ }^{4}$ ), Éthier-Blais, donc, aura malgré tout donné rendez-vous à ses lecteurs francophones dans un quart de siècle. Il aura fait un pari sur l'avenir. Le sien, d'abord. Et aussi celui de cette littérature dont il est une incarnation exemplaire et qu'il a si bien servie.

1. Cabiers Ëthier-Blais. Documents - Essais Outils - Témoignages, automne 1998, $n^{\circ}$ 1, Ottawa, Le Nordir, 114 p. Je souligne d'emblée que j'y ai mon nom, mais comme membre du "Comité de patronagen, fonction honorifique qui m'est échue, je ne sais trop comment. Comme je n'ai participé ni à la gestation de ce projet ni à sa réalisation, je n'ai aucun scrupule à en parler dans cette chronique.

2. Martin Doré (dir.), Jean Étbier-Blais: une vie en écriture, Montréal, Hurtubise $\mathrm{HMH}$, 1997, 204 p.

3. Martin Doré et de jean-Pierre Duquette (dir.), Jean Étbier-Blais: dictionnaire de lui-même. Essais sur l'œuvne, Montréal, Fides, 1998, $324 \mathrm{p}$.

4. Jean Éthier-Blais, Dictionnaire de moimême, Montréal, Leméac, 1987, p. 99. 